\title{
Improving Access to Bariatric Surgery for Rural and Remote Patients: Experiences from a State-Wide Bariatric Telehealth Service in Australia
}

\author{
Andrew Phillip Maurice ${ }^{1,2}$ (D) Jaisil Eldo Joseph Punnasseril ${ }^{1,2} \cdot$ Sarah Emily King ${ }^{1,2} \cdot$ Benjamin Rees Dodd $^{1,2}$
}

Received: 24 March 2020 / Revised: 8 June 2020 / Accepted: 18 June 2020 / Published online: 2 July 2020

(C) Springer Science+Business Media, LLC, part of Springer Nature 2020

\begin{abstract}
Background The state of Queensland, Australia, is large $\left(1.85\right.$ million $\left.\mathrm{km}^{2}\right)$. The provision of bariatric care across the state is difficult as most major hospitals are concentrated in the capital city of Brisbane. We implemented a state-wide telehealth service to improve access for rural patients in a public bariatric service. We report our early experiences with this service.

Methods We reviewed all patients seen in the Bariatric Telehealth Clinic from 2017 to 2019. Patients underwent consultation through video-link software at their local hospital with the multidisciplinary team in Brisbane (surgeon, dietician and clinical nurse). Distances from Brisbane and number of visits were calculated. Telehealth patients were contacted by phone to complete a survey regarding their experiences. This was a 17-question Likert-style survey with scores from 1 (strongly disagree) to 5 (strongly agree).

Results A total of 85 new patients underwent their initial consultation via telehealth. Each patient had a variable number of inperson as well as telehealth consultations both before and after surgery. Mean distance from telehealth consultation site to Brisbane was $614 \mathrm{~km}$ (range 149-2472 km). In total, 41 (48\%) completed the survey. With regard to telehealth saving time and money, improving access to bariatric care, and desire to use telehealth again, the mean score was 4 out of 5 or higher for all questions (i.e., agree or strongly agree). There was no identifiable post-operative complication that was caused or exacerbated by telehealth.

Conclusion Bariatric surgical telehealth appointments are feasible and preferred by most patients residing in rural and remote locations. Each consultation avoids significant travel time and cost for the patient and health service, with no obvious adverse outcomes. Telehealth improves equity and access to specialist services for rural and remote patients.
\end{abstract}

Keywords Telehealth $\cdot$ Telemedicine $\cdot$ Bariatric surgery $\cdot$ Remote $\cdot$ Rural

\section{Introduction}

For patients who are obese, bariatric surgery is a highly effective therapy for weight loss and obesity-related comorbidities [1]. Care of the bariatric patient is complex and requires multidisciplinary pre-operative evaluation and regular post-

Andrew Phillip Maurice

andrew.maurice@uqconnect.edu.au

1 Department of Upper Gastrointestinal and Bariatric Surgery, Royal Brisbane and Women's Hospital, Brisbane, Queensland, Australia

2 Discipline of Surgery, School of Medicine, The University of Queensland, Brisbane, Queensland, Australia operative review. Australian and International guidelines recommend bariatric surgery should only be offered in high-volume, specialized units with adequate allied-health support and follow-up capability $[2,3]$. This is required to prevent late weight regain and avoidable complications such as nutritional deficiencies [4, 5]. Increased patient geographical distances from bariatric units are associated with poorer follow-up and potentially poorer outcomes [6-8].

Most high-volume bariatric practices in Australia are based in large, metropolitan centers. Australia is the 7th most sparsely populated country in the world, with an area of over 7.5 million $\mathrm{km}^{2}$ (31 times the size of the UK) and a population of 25 million. Access to medical services is challenging for patients in regional and remote areas [9], especially for the bariatric patient who requires a multidisciplinary team. 
Telehealth services have been developed in a variety of settings in medicine to assist with the delivery of health care services to regional and remote patients $[10,11]$. These services typically involve a videoconference in which the physician at a tertiary hospital can interview and review a patient at a remote health care site (i.e., at their local General Practitioner (GP) or hospital). There is limited published data regarding the use of telehealth in bariatric surgery. The recent COVID19 outbreak has dramatically increased the interest, demand, and development of telehealth services [12], including in surgery $[13,14]$.

We implemented a state-wide bariatric telehealth service in the large state of Queensland, Australia. We describe our experiences with the delivery of pre-operative and postoperative consultations through telehealth and have performed a patient satisfaction survey.

\section{Methods}

\section{Setting}

Australia has a hybrid health system in which approximately half of the population are privately insured and the remainder are treated in the public (i.e., tax-payer funded) healthcare system [15]. The majority ( $88 \%$ ) of bariatric surgery is performed in the private system in Australia [16]. The Royal Brisbane and Women's Hospital (RBWH) in Brisbane, Queensland, Australia has provided a high-volume, multidisciplinary bariatric service since 2000. This is a tax-payer funded, public service run by the Government in a public hospital.
Standard post-operative follow-up for our patients include a clinical nurse phone call at 1 week, initial clinic attendance at 6-8 weeks, then every 3 months for the first year and at least every 6 months for up to 5 years. Each patient is assessed by a multidisciplinary team at each consultation. The team consists of a bariatric surgeon, bariatric dietician, and clinical nurse. A psychologist is employed selectively. Routine blood tests are performed at least 6-monthly to assess for nutritional deficiencies. The state of Queensland is large $\left(1.85\right.$ million $\left.\mathrm{km}^{2}\right)$ with a population of 5 million and a low population density of $2.92 /$ $\mathrm{km}^{2}$. The vast majority of the population and health care services are located in the south-east of the state, in or near the capital city of Brisbane.

\section{Bariatric Telehealth Service}

In September 2017, RBWH began offering telehealth appointments for new and review bariatric patients for those outside of Brisbane. The telehealth clinic is conducted once per month, in addition to two other traditional in-person bariatric surgery clinics each month. This involves a videoconference with each member of the multidisciplinary team located at the RBWH clinic (Fig. 1). The patient would attend their local General Practitioner or remote/regional hospital for the telehealth consultation. Patient eligibility for this service was assessed on a case-by-case basis provided the bariatric service staff, patient, and local hospital all agreed that such consultations would be feasible and desirable. In addition to any telehealth appointments before and after surgery, all patients had at least one in-person traditional clinic appointment before and after their surgery. Post-operative telehealth appointments
Fig. 1 Telehealth consultation. Telehealth consultations are provided via video-link through the patient's local hospital or general practitioner office. The surgeon (pictured) or multidisciplinary team member will consult with the patient individually (pictured), or in a group setting if appropriate






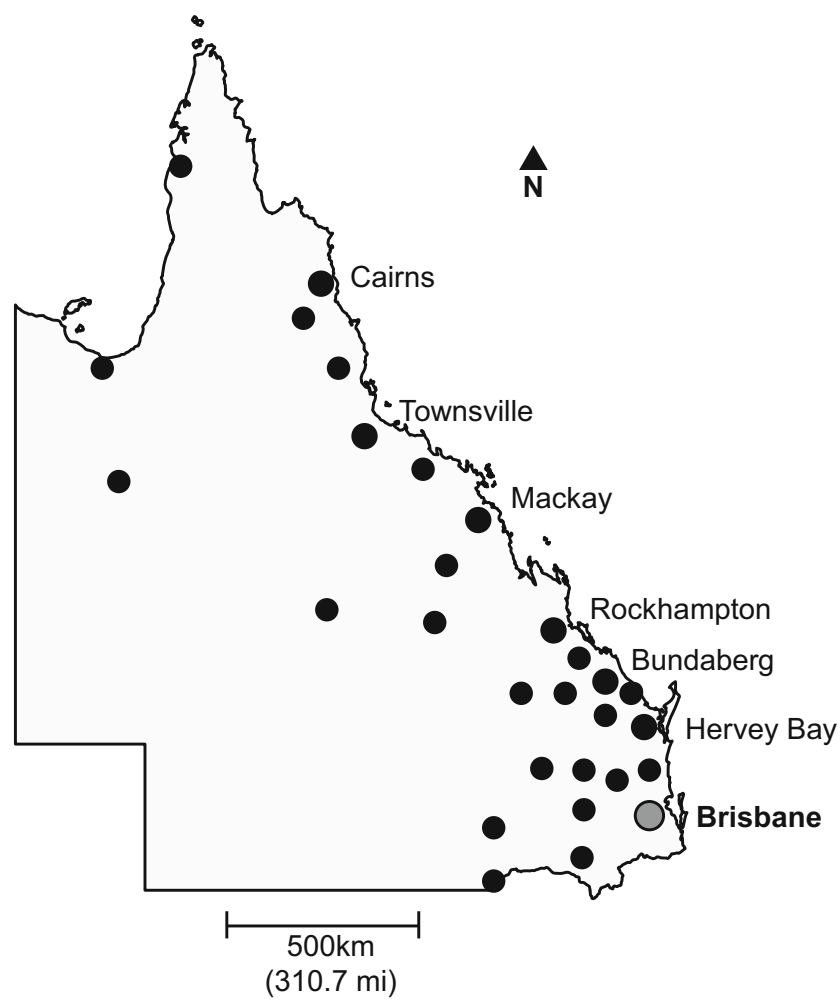

Fig. 2 Map of the state of Queensland, Australia. Each dot corresponds to a town/locale from which a patient consulted the bariatric telehealth service. Some towns had multiple patients referred. Selected large regional towns are listed. The location of the RBWH is in Brisbane (gray circle, south-east). The scale demonstrates the large size of the state

were offered provided all parties agreed to this. This meant that if the surgeon, patient or local physician were concerned, an in-person appointment would be conducted. In practice, all patients had a combination of both in-person and telehealth post-operative appointments.

\section{Data Collection and Review}

A prospective database of all patients who had used the service was accessed to identify patients. A retrospective review of all patients who had utilized the bariatric telehealth services at least once pre-operatively was undertaken (i.e., "new" patients). Medical records were reviewed for basic patient demographics and geographic location, including those who never progressed to surgery. The number of pre-operative and post-operative in-person and telehealth appointments was recorded. The distance and approximate driving time from the patient's home address to the RBWH was calculated through Google Maps (Fig. 2).

A telephone questionnaire was conducted to assess patient satisfaction with the telehealth service. These questions and results are presented in Figs. 3, 4, 5, 6, and 7. Similar Likertstyle questionnaires have been used effectively in a variety of medical and surgical telehealth surveys to accurately capture patient satisfaction and our survey mirrors these [17-20]. A series of statements were read to the participant, and they were asked to rate their response on a scale from 1 (strongly disagree) to 5 (strongly agree). The questionnaire covered five aspects of telemedicine: satisfaction with travel benefits, equipment and technical issues, clinical interaction, communication, and future considerations. The survey was concluded by allowing the patients to provide free additional comments about their experience with the service.

Post-operative complications following discharge were identified from the patient record and checked at time of phone questionnaire and were graded according to the Clavien-Dindo scale [21]. State-wide public health records were searched to ensure presentations to other hospitals were not missed. In-hospital complications related to surgery were not analyzed as these were considered

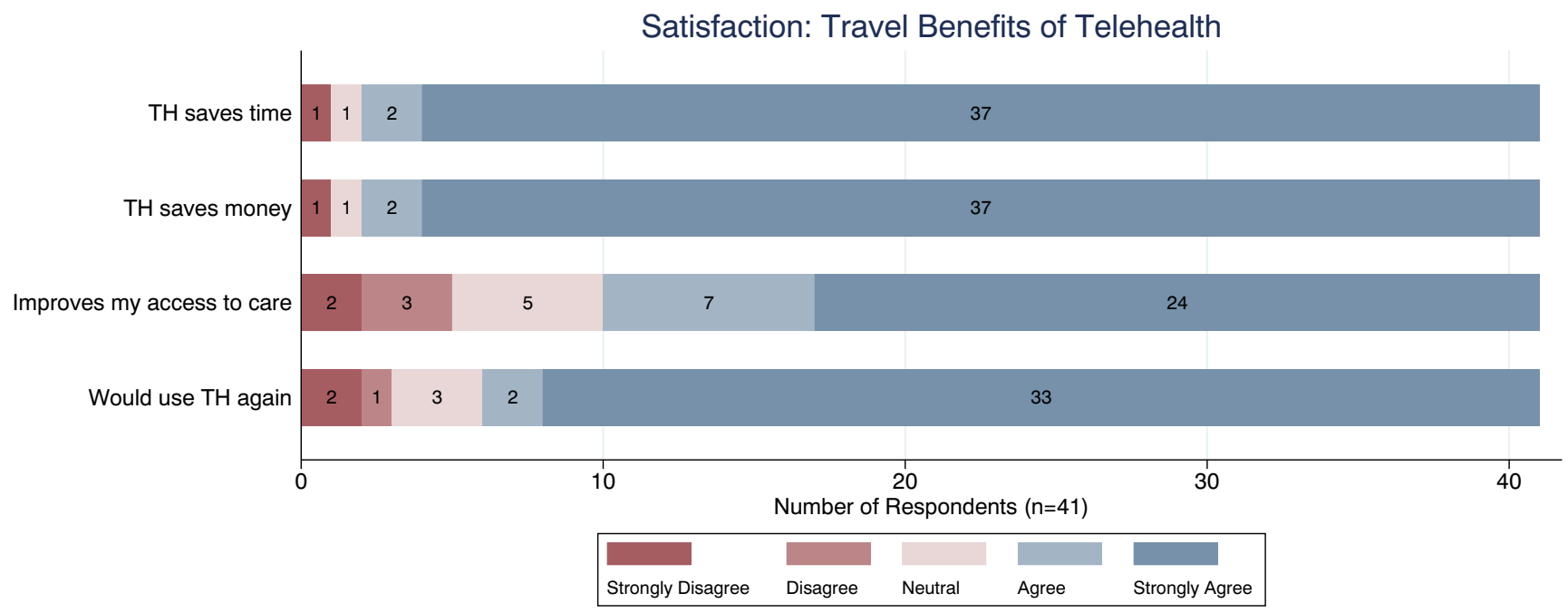

Fig. 3 Survey results for travel benefits. The patients felt strongly that the telehealth program was cost-saving and convenient (TH: Telehealth) 


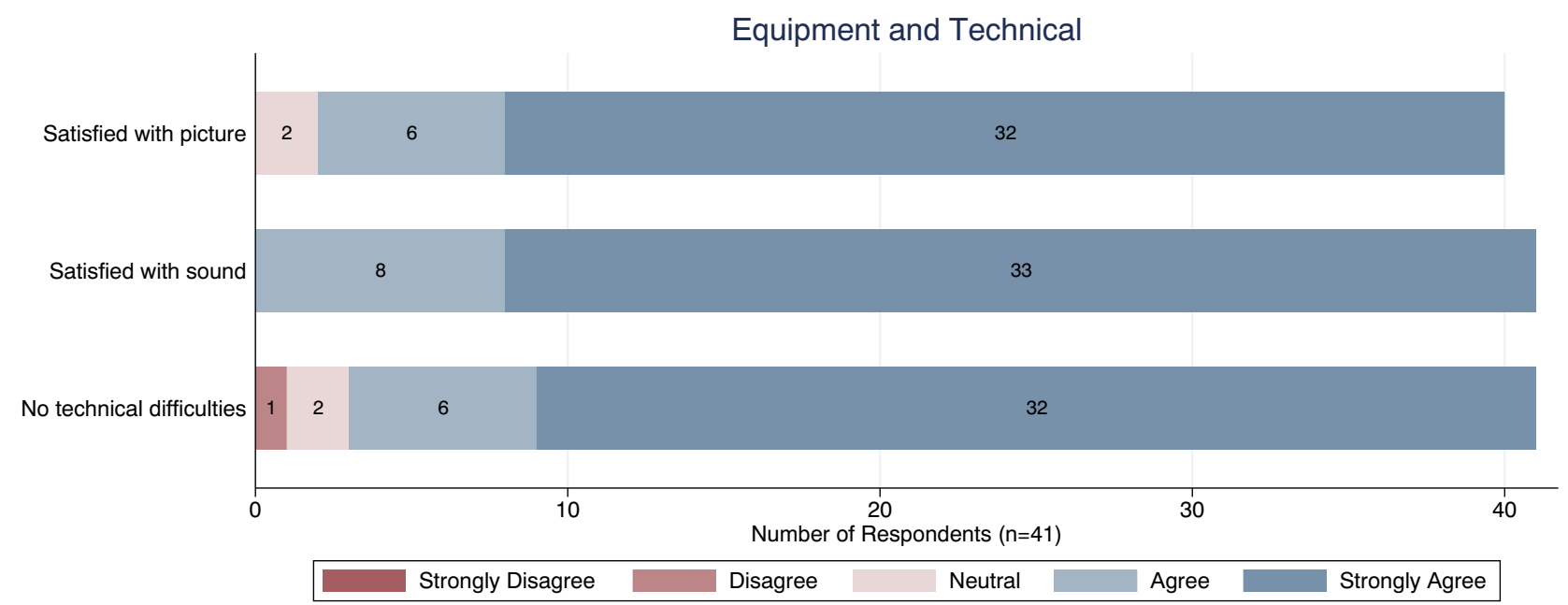

Fig. 4 Survey results regarding equipment and technical aspects of the consultation. There were minimal technical difficulties with the telehealth program reported by patients

irrelevant to the telehealth service appraisal. Weight loss and comorbidity resolution outcomes were not assessed as part of this study.

Ethical approval was sought and obtained from the local hospital ethics board. All procedures performed in studies involving human participants were in accordance with the ethical standards of the institutional and/or national research committee and with the 1964 Helsinki declaration and its later amendments or comparable ethical standards. Informed consent was obtained from each patient to conduct the phone survey.

\section{Statistics}

This is a descriptive study and the results have been presented as summary descriptive statistics. Figures and summary statistics were generated using STATA version 15.1. As a qualitative study with no comparison cohort, no statistical tests have been performed.

\section{Results}

There were 85 patients identified. All 85 patients underwent their initial consultation through the telehealth service. The mean age of the patients was 47.6 years (range 21-73 years) and 54 (64\%) were female. The mean distance ( \pm 1 standard deviation) from the RBWH to the local telehealth site, as estimated by Google Maps, was $614 \pm 516 \mathrm{~km}$ (range 149-2472 km). This corresponded to a mean ( \pm 1 standard deviation) driving time of $6.60 \pm$ $4.84 \mathrm{~h}$ (range 1.93-19.75 h). A map indicating the

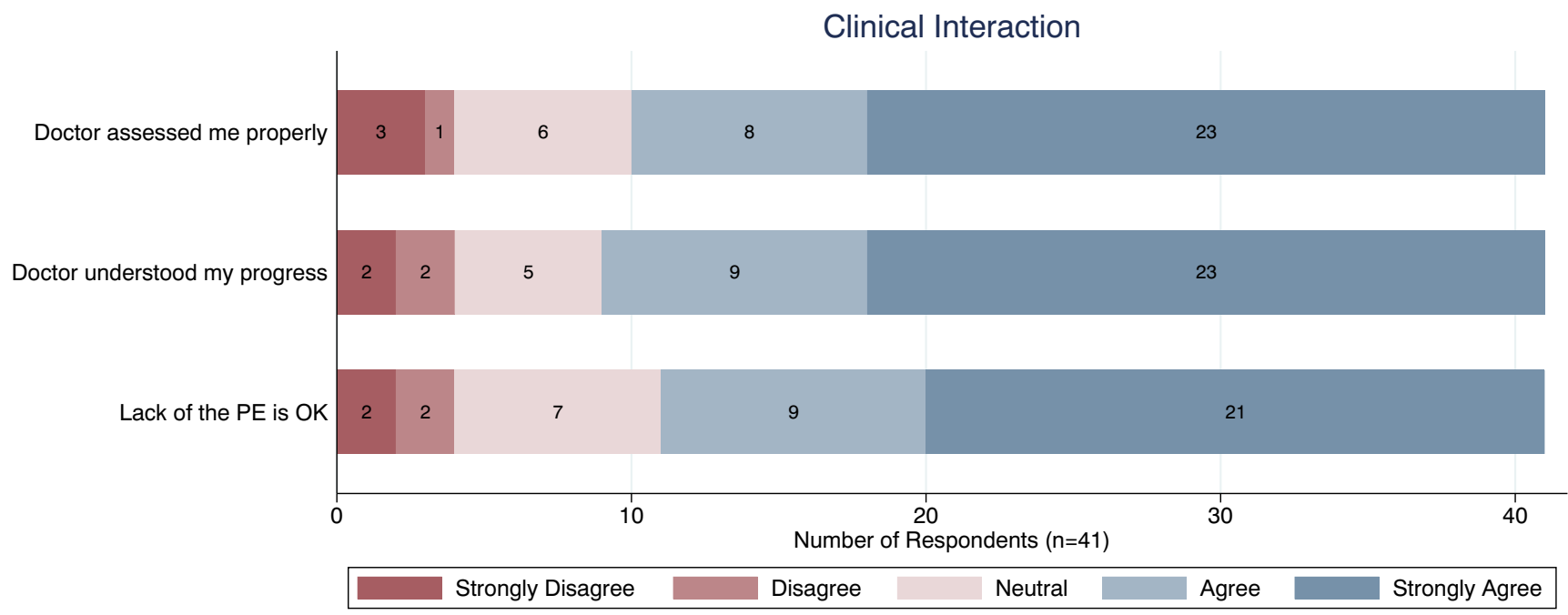

Fig. 5 Survey results regarding the clinical interaction and perceptions of doctor interaction. Patients were mostly satisfied with the interaction with the doctor (PE: physical examination) 


\section{Communication and Rapport}

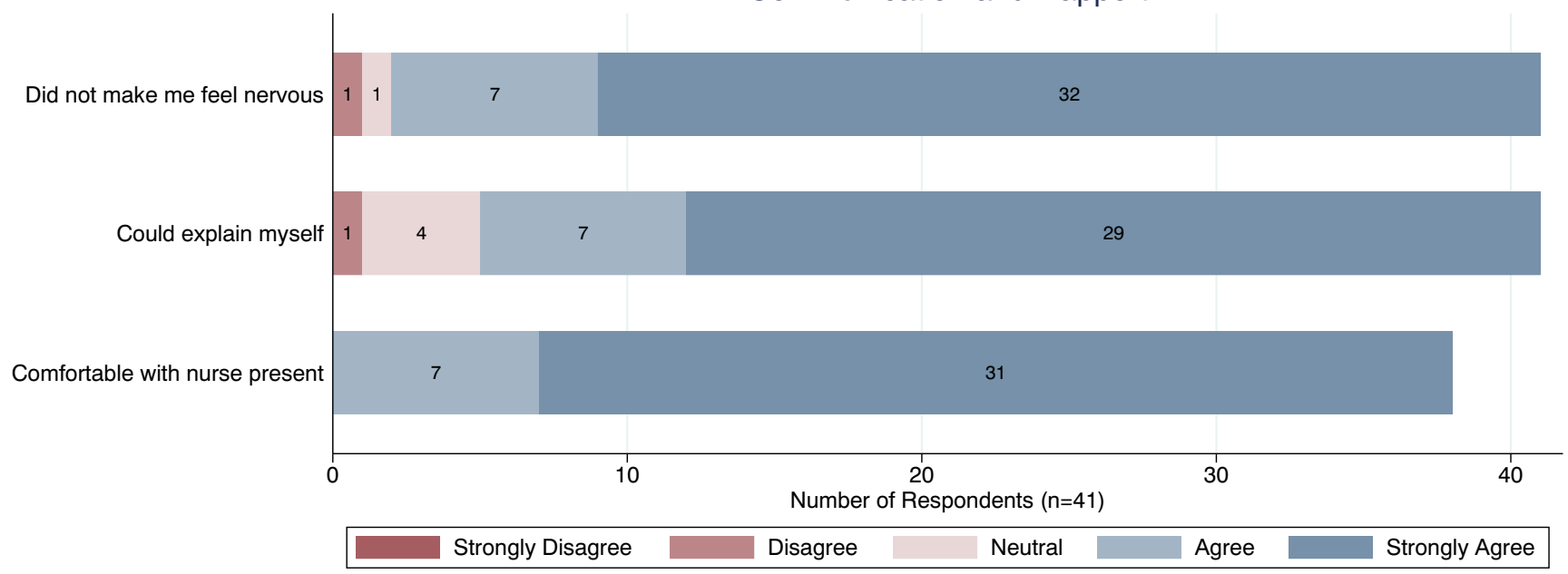

Fig. 6 Survey results regarding communication and rapport. There are less than 41 respondents for feelings regarding having a nurse present as

some patients only had the local doctor (without nurse present) for the consultation

location of each patient within the state in reference to the RBWH is given in Fig. 2.

\section{Patients Who Received Surgery}

Of the 85 patients, 52 progressed to surgery. Twenty underwent laparoscopic Roux-en-Y gastric bypass (RYGB), 19 underwent sleeve gastrectomy, and 11 underwent singleanastomosis (One-anastomosis) gastric bypass. Two patients underwent laparoscopy where the planned procedure was aborted for technical reasons (specifically, inadequate liver shrinkage and poor access). One patient suffered an inhospital complication and died before discharge.

A detailed overview of all recorded post-discharge complications is given in Table 1. No Clavien-Dindo grade 4 (complication resulting in organ failure) or 5 (death) complications were detected. There were 5 Clavien-Dindo Grade $3 b$ complications (re-operations), two of which were performed emergently and three were elective revisions. One of these (laparotomy and adhesiolysis for small bowel obstruction) occurred within days of discharge. The three elective revisions were performed after the patients noted complications at the telehealth visits (intractable reflux or dysphagia). Three patients required an endoscopy (one of which had new iron deficiency caused by peri-stomal ulceration) and two patients were diagnosed with nutritional deficiencies that responded to treatments provided by the local general practitioner (parenteral B12 and iron infusion).

For those that had progressed to surgery, the average number of pre-operative telehealth consultations was 1.2 (range 1-

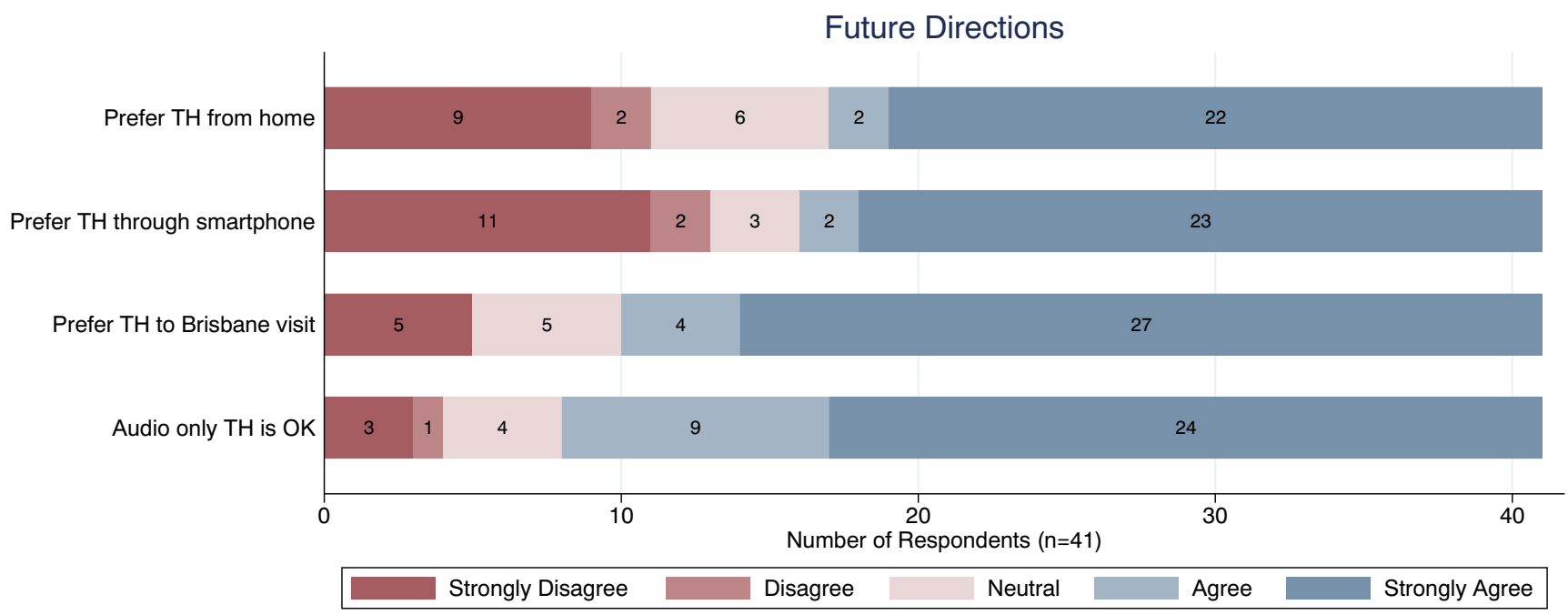

Fig. 7 Survey results assessing future directions for the telehealth service. There was hesitation from several patients about potential changes to the telehealth visit. Most patients expressed a desire to continue visits to the local hospital, rather than have home-telehealth consultations (TH: Telehealth) 


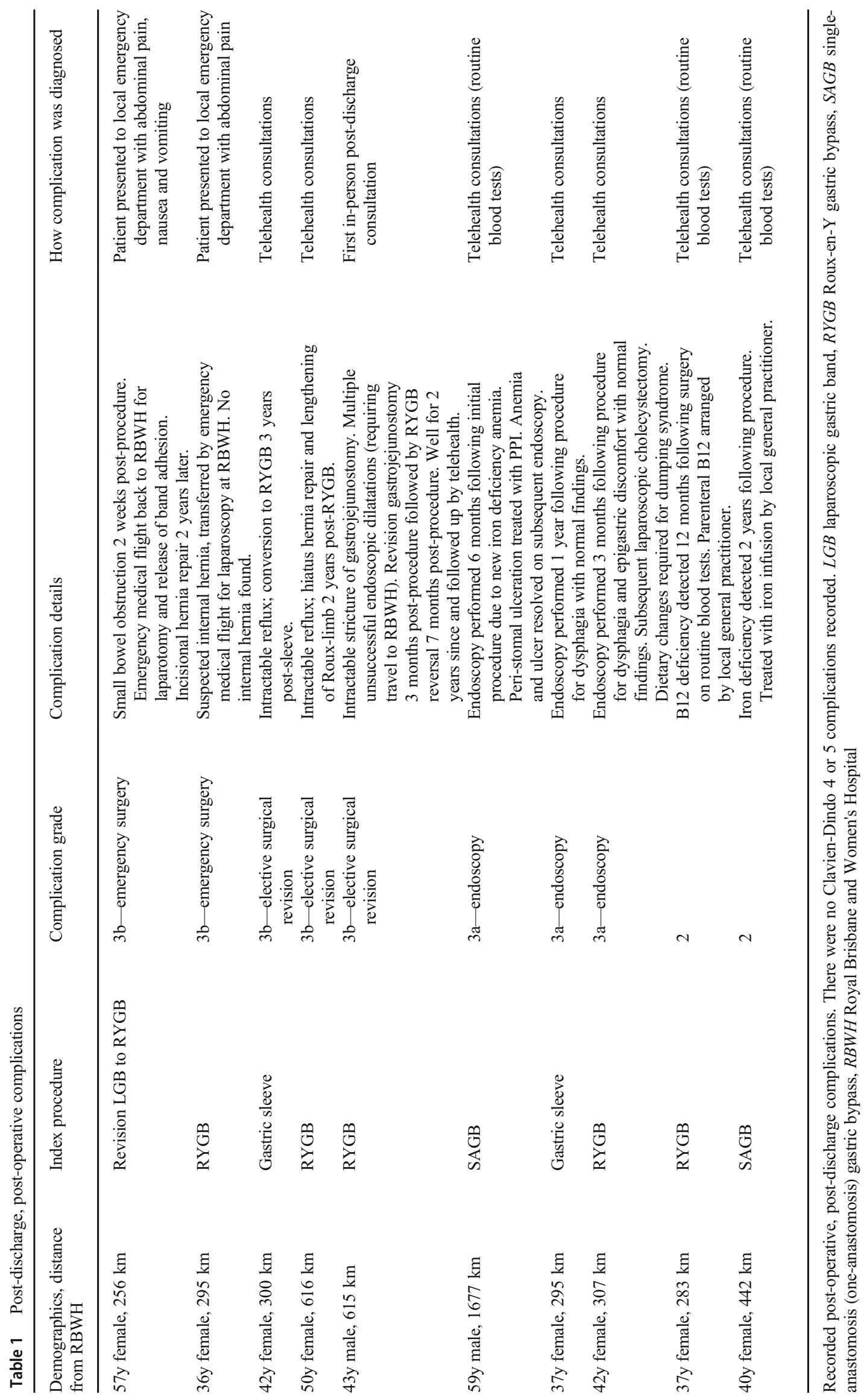


4). Forty patients had only one pre-operative telehealth appointment. The average number of pre-operative in-person appointments was 1.59 (range 1-3). All patients that required more than one in-person or one telehealth pre-operative appointment had medical conditions requiring evaluation and optimization prior to surgery (e.g., diabetes management or need to obtain pre-operative investigations such as echocardiograms).

The mean number of post-operative appointments by telehealth for each patient was 2.8 (range 0 to 13) and the mean number of post-operative in-person appointments was 2.5 (range 1 to 6). The mean ratio of post-operative telehealth to in-person appointments was 1.58:1 (range 0-6.5, i.e., on average, for each in-person post-operative appointment, each patient had 1.58 telehealth appointments).

Of the patients that received surgery, nine (17\%) were eventually lost to follow-up (i.e., minimum three attempts to contact patient). RBWH and state-wide electronic medical records did not reveal any post-operative complications in these patients attributable to their bariatric surgery (this does not account for private hospital or interstate healthcare encounters). For those who eventually were lost to follow-up, the mean number of in-person post-operative appointments was 1.89 (range 1-4) and the mean number of telehealth post-operative appointments was 1.56 (range $0-4$ ).

\section{Patients Who Have Not Received Surgery}

Of the 32 patients who have not yet received surgery, six are on the surgical waiting list and two are undergoing preoperative investigations at the time of publication. Two patients were able to lose weight to achieve a BMI of less than 35 and were no longer considered for surgery. Five patients opted to not pursue surgery and did not attend RBWH in-person. Eleven patients were lost to follow-up after initial bariatric telehealth appointment. One patient died of a sleep apnearelated illness and five patients were diagnosed preoperatively with a serious malignancy and were no longer deemed suitable for surgery.

\section{Phone Survey Results}

Survey results were obtained in $41(48 \%)$ patients. The results of the phone survey are given in Figs. 3, 4, 5, 6, and 7. Patients were able to provide free comments about their experience and a selection of representative quotes is provided in the Appendix. The patients described a high degree of satisfaction with the service, indicating strongly that it saved time and money for almost all of them. There was no marked dissatisfaction with the technical quality of telehealth consultation.

Patients expressed some resistance to the proposed changes in the survey. Specifically, the patients consistently stated that visiting their local hospital or doctor's office, with in-person assessment, was preferable to telehealth provided solely from home (e.g., through Skype). Many patients also stated that occasional in-person visits to the traditional bariatric clinic at RBWH was necessary and desirable.

Two patients expressed "strongly disagree" for virtually all questions. Both of these patients experienced significant complications with their surgery. A different patient expressed that the telehealth service did not allow them to convey their reflux severity to the clinicians (see comment in Appendix).

\section{Discussion}

This study documents our early experiences with delivering a bariatric telehealth service from initial consultation through to post-operative care at a high-volume, state-wide public hospital service over a large geographical area. Our experience demonstrates this is feasible with no associated major morbidity. There are clear potential cost savings to both the health service and to individual patients. A high degree of patient satisfaction was evident in the survey results.

There are few studies that describe bariatric telehealth delivery to remote patients. A 2018 systematic review of the use of telehealth services in bariatric surgery (not specific to remote patients) identified 10 manuscripts, 5 of which were small feasibility studies [22]. The primary focus of most of the studies was education and behavioral change (rather than follow-up for medical issues), which was delivered through online modules [23], telephone calls [24], or videoconferencing [25]. One feasibility study examined the delivery of postoperative consultations through videoconferencing [26]. Another study examined the use of telehealth with mobile devices and telephone calls to monitor patient progress (e.g., weight loss) as an adjunct to traditional consultations [27]. Whilst outcomes and success were mixed for these studies, all reported a high rate of patient satisfaction.

A subsequent 2019 study of the provision of telehealth bariatric services in rural Ontario, Canada, examined 96 patients who had post-operative telehealth review and compared these with 96 matched patients who underwent traditional inperson post-operative consultations [28]. There was no statistically significant difference in 2-year loss to follow-up rates comparing the telehealth consultations to the in-person consultations. No clinical outcomes or patient satisfaction data were reported. The authors concluded that telehealth services can facilitate post-operative follow-up in rural patients. To our knowledge, our study is the only other similar study to date describing the delivery of bariatric surgery telehealth consultations as a solution to patient remoteness.

Although a formal economic analysis has not been performed in this study, it is clear that the bariatric telehealth service likely saves a significant amount of money for the patient and health system. Prior to 2017, all patients were 
flown to Brisbane for all pre-operative and post-operative appointments. Patients are only partially reimbursed for these costs. Most patients do not have any significant postoperative concerns or issues, yet require flights, and often overnight accommodation, to see the multidisciplinary team for less than an hour. Such a clinic visit consumes one to two full days of each patient's time. It is consistent in the formal survey and free comments that telehealth reduced this significant inconvenience for patients. The cost saving aspect of telehealth with respect to flights in other areas of medicine has been reported by others in Australia [29].

Telehealth is an important consideration in delivering equity of access in bariatric surgery and medicine in general, especially given that the RBWH is a publicly funded service. The lack of access to high-quality specialist healthcare in rural and remote sites is an ethical and social-justice issue [30] and the provision of bariatric services is no exception. The recent COVID-19 outbreak has brought many of the above issues into sharp focus [13]. The epidemic has already forced many surgical practices to implement or dramatically increase their telehealth services and such transitions can be problematic [31]. Whether the physicians approve or not, it is likely usage of telehealth in surgery will only increase in the long term due to COVID-19 and a "silver-lining" will be the rapid improvement of telehealth services globally [32, 33].

Telehealth has significant potential benefits in the preoperative setting. In this study, several patients were initially consulted and declined surgery. In addition, several patients were able to lose weight and avoided surgery. It is likely that the telehealth services avoided considerable expense and inconvenience for these patients who, prior to 2017 when the telehealth service started, would have had to travel to RBWH at least once for an in-person consultation. Furthermore, the telehealth services allowed identification of pre-operative issues that could be investigated and managed locally, prior to in-person attendance (e.g., sleep study and CPAP prescription).

Of particular interest are the 11 patients that were initially consulted via telehealth but did not attend further appointments and were lost to follow-up. In addition to the saved expenditure for avoiding travel for patients who ultimately did not proceed with surgery, our anecdotal experience suggests that pre-operative non-compliance and non-attendance may correlate with poor outcomes and future behaviors. There are numerous studies detailing the link between post-operative attendance with improved outcomes [34-36]; however, there is a paucity of literature investigating the effect of pre-operative noncompliance. Several small retrospective studies have demonstrated that poor pre-operative clinic attendance is associated with poorer bariatric outcomes $[37,38]$. Further formal studies examining the effect of pre-operative attendance with outcomes would be warranted.
There are potential caveats to a telehealth service. The most significant is missed morbidity that may have been detected through an in-person consultation. The most serious recorded complications were two emergent re-operations and three elective re-operations (Clavien-Dindo 3b). It does not appear that telehealth caused or exacerbated morbidity from these presentations; however, we have provided details in Table 1 for each complication such that the reader can evaluate these. It is unlikely the two emergent re-operations (re-laparoscopy for suspected internal hernia and laparotomy for bowel obstruction) would be related to the telehealth service. Two patients were diagnosed with nutritional deficiencies and were successfully treated by the local General Practitioner (iron infusion and parenteral B12). In contrast, we believe this demonstrates the success of the program: that nutritional deficiencies can be monitored and treated remotely.

In the survey, one patient commented that they felt their post-operative reflux was being ignored and perhaps this would be addressed better in an in-person consultation. The patients consistently stated that they preferred at least some of their post-operative appointments to be conducted in-person. Furthermore, some patients were uncomfortable with the potential for future telehealth consultations to be performed at home. There was a clear patient preference for attendance at their local hospital or family doctor for telehealth appointments and it was felt that the local doctor would be able to provide additional post-operative surveillance and support. We hypothesize that it may be easier to inappropriately "ignore" or downplay a patients concern in the telehealth format compared to an in-person consultation. There is surprisingly little literature published regarding this phenomenon.

This study is limited by its retrospective nature, losses to follow-up, and small number of patients assessed. For some respondents, it had been months since their last telehealth or in-person appointment, potentially introducing recall bias. We did not compare the telehealth patients to the remainder of the patients seen in the traditional in-person clinic. As with any retrospective study or survey, there will be losses-to-follow up, refusals to participate and patients who do not answer the phone call. These patients may have an important outcome of interest to this study (e.g., dissatisfaction with the service, an adverse outcome or some other relevant information) and are a further source of potential bias. Weight loss outcomes have not been reported in this study as it is difficult to correlate weight loss outcomes to the telehealth service in the presence of significant confounders. To improve detection of complications for those that did not participate in the survey, we used state-wide medical records to ensure the patients did not present to different hospitals with complications; however, this would have not included patients seen in private facilities or who have moved interstate.

There are several important issues in which future research in this area should focus on. Other bariatric services with a 
telehealth component (particularly for those with a focus on rural and remote patients) should report their experiences, especially with respect to feasibility, modalities of delivery, patient satisfaction, modalities of funding (private insurance versus government funding), and potential pitfalls as there are very few reports published to date. A formal cost-benefit analysis would be useful to perform. The use of novel telehealth modalities (e.g., phone-apps) have been reported in bariatric telehealth [10] and these should be investigated further. Telehealth modalities should be assessed for their impact in reducing loss to follow-up and improving patient compliance with treatment plans. Finally, although our service is public (i.e., tax-payer funded), the majority of bariatric surgery in Australia is performed in private practice. The role of telemedicine and issues of remote medicine in general in private practice environments (e.g., the USA) requires further investigation.

\section{Conclusions}

Bariatric telehealth for rural and remote patients is feasible and associated with a high degree of patient satisfaction. It is likely such a service saves a significant amount of time, inconvenience, and expense for both the patient and health service. There were no obvious adverse effects or patient harm associated with the telehealth service. Telehealth is an important tool to improve equity and access to high-quality specialist services for rural and remote patients.

Acknowledgements The RBWH telehealth service is facilitated by the hard work of clinical nurses Zoe Jarrott and Jess Donnan and clinical practice coordinator Kath Bradley. The patients and surgeons are indebted to the hard work and effort from these outstanding individuals.

Funding COIs have been completed for each author. All authors have declared no conflict of interests regarding the research and publication of this article and have no relevant disclosures.

\section{Compliance with Ethical Standards}

Blinded COI/Ethics/Consent Statements Andrew Maurice (Author 1) declares no conflict of interest.

Jaisil Punnasseril (Author 2) declares no conflict of interest.

Sarah King (Author 3) declares no conflict of interest.

Benjamin Dodd (Author 4) 4 declares no conflict of interest.

Signed un-blinded COI statements have been submitted as part of the manuscript submission separately as per journal guidelines.

\section{Appendix: Selected Representative Patient Comments from Survey}

"Very impressed with the long-term care. Feels like I haven't been forgotten."
"I would much rather travel further for a 10 minute face-toface appointment despite the travel and cost. I have had reflux since the surgery but the telehealth service has not provided adequate care for this problem."

"Helps save so much travel time, but doctors didn't know much about my background or medical history. Inconsistency of doctors is problematic."

"The service is fantastic and makes everything so easy and quick."

\section{References}

1. Nguyen NT, Varela JE. Bariatric surgery for obesity and metabolic disorders: state of the art. Nat Rev Gastroenterol Hepatol. 2017;14(3):160-9.

2. Busetto L, Dicker D, Azran C, et al. Practical recommendations of the obesity management task force of the European Association for the Study of obesity for the post-bariatric surgery medical management. Obes Facts. 2017;10(6):597-632.

3. Council NHaMR. Clinical Practice guidelines for the management of overweight and obesity in adults, adolescents and children in Australia; 2013.

4. Lim R, Beekley A, Johnson DC, et al. Early and late complications of bariatric operation. Trauma Surg Acute Care Open. 2018;3(1): e000219.

5. Ziegler O, Sirveaux MA, Brunaud L, et al. Medical follow up after bariatric surgery: nutritional and drug issues. General recommendations for the prevention and treatment of nutritional deficiencies. Diabetes Metab. 2009;35(6 Pt 2):544-57.

6. Mehaffey JH, Michaels AD, Mullen MG, et al. Patient travel for bariatric surgery: does distance matter? Surg Obes Relat Dis. 2017;13(12):2027-31.

7. Jennings N, Boyle M, Mahawar K, et al. The effect of distance from the bariatric Centre on follow up compliance and possible consequences for weight loss in a UK population: B04. Br J Surg. 2012;99

8. Sivagnanam P, Rhodes M. The importance of follow-up and distance from centre in weight loss after laparoscopic adjustable gastric banding. Surg Endosc. 2010;24(10):2432-8.

9. Bourke L, Humphreys JS, Wakerman J, et al. Understanding rural and remote health: a framework for analysis in Australia. Health Place. 2012;18(3):496-503.

10. Bradford NK, Caffery LJ, Smith AC. Telehealth services in rural and remote Australia: a systematic review of models of care and factors influencing success and sustainability. Rural Remote Health. 2016;16(4):3808.

11. Ekeland AG, Bowes A, Flottorp S. Effectiveness of telemedicine: a systematic review of reviews. Int J Med Inform. 2010;79(11):73671.

12. Portnoy J, Waller M, Elliott T. Telemedicine in the era of COVID19. The Journal of Allergy and Clinical Immunology: In Practice. 2020.

13. Hakim AA, Kellish AS, Atabek U, et al. Implications for the use of telehealth in surgical patients during the COVID-19 pandemic: Elsevier; 2020.

14. Loeb AE, Rao SS, Ficke JR, et al. Departmental experience and lessons learned with accelerated introduction of telemedicine during the COVID-19 crisis. J Am Acad Orthop Surg. 2020;28:e46976. 
15. Collyer F, Willis K, Keleher H. The private health sector and private health insurance. Understanding the Australian Health Care System. 2019:37.

16. Health AIo, Welfare. Weight Loss Surgery in Australia 2014-15. Canberra: Australian Hospital Statistics; 2017.

17. Shivji S, Metcalfe P, Khan A, et al. Pediatric surgery telehealth: patient and clinician satisfaction. Pediatr Surg Int. 2011;27(5):523-6.

18. Xu T, Pujara S, Sutton $\mathrm{S}$, et al. Peer reviewed: telemedicine in the management of type 1 diabetes. Prev Chronic Dis. 2018;15

19. Morgan DG, Kosteniuk J, Stewart N, et al. The telehealth satisfaction scale: reliability, validity, and satisfaction with telehealth in a rural memory clinic population. Telemed e-Health. 2014;20(11): 997-1003.

20. Agha Z, Schapira RM, Laud PW, et al. Patient satisfaction with physician-patient communication during telemedicine. Telemed e-Health. 2009;15(9):830-9.

21. Dindo D. The Clavien-Dindo classification of surgical complications. Treatment of postoperative complications after digestive surgery: Springer; 2014. p. 13-7.

22. Coldebella B, Armfield NR, Bambling M, et al. The use of telemedicine for delivering healthcare to bariatric surgery patients: a literature review. J Telemed Telecare. 2018;24(10):651-60.

23. Bradley LE, Thomas JG, Hood MM, et al. Remote assessments and behavioral interventions in post-bariatric surgery patients. Surg Obes Relat Dis. 2018;14(10):1632-44.

24. Sudan R, Salter M, Lynch T, et al. Bariatric surgery using a network and teleconferencing to serve remote patients in the Veterans Administration Health Care System: feasibility and results. Am J Surg. 2011;202(1):71-6.

25. Baillot A, Boissy $\mathrm{P}$, Tousignant $\mathrm{M}$, et al. Feasibility and effect of inhome physical exercise training delivered via telehealth before bariatric surgery. J Telemed Telecare. 2017;23(5):529-35.

26. Morrow E, Bruce DM, Bruce E, et al. Post surgical review of bariatric surgery patients: a feasibility study of multidisciplinary follow up using videoconferencing. Clin Pract Epidemiol Ment Health. 2011;7:84-8.

27. Vilallonga R, Lecube A, Fort JM, et al. Internet of things and bariatric surgery follow-up: comparative study of standard and IoT follow-up. Minim Invasive Ther Allied Technol. 2013;22(5):304 11.

28. Wang CD, Rajaratnam T, Stall B, et al. Exploring the effects of telemedicine on bariatric surgery follow-up: a matched case control study. Obes Surg. 2019;29:2704-6.

29. Thaker DA, Monypenny R, Olver I, et al. Cost savings from a telemedicine model of care in northern Queensland, Australia. Med J Aust. 2013;199(6):414-7.

30. Fleming DA, Edison KE, Pak H. Telehealth ethics. Telemed eHealth. 2009;15(8):797-803.

31. Grenda TR. Transitioning a Surgery Practice to Telehealth During COVID-19.

32. Keesara S, Jonas A, Schulman K. Covid-19 and health care's digital revolution. N Engl J Med. 2020;382:e82.

33. Wosik J, Fudim M, Cameron B, et al. Telehealth transformation: COVID-19 and the rise of virtual care. J Am Med Inform Assoc. 2020;27:957-62.

34. Sockalingam S, Cassin S, Hawa R, et al. Predictors of post-bariatric surgery appointment attendance: the role of relationship style. Obes Surg. 2013;23(12):2026-32.

35. Moroshko I, Brennan L, O'Brien P. Predictors of attrition in bariatric aftercare: a systematic review of the literature. Obes Surg. 2012;22(10):1640-7.

36. Compher CW, Hanlon A, Kang Y, et al. Attendance at clinical visits predicts weight loss after gastric bypass surgery. Obes Surg. 2012;22(6):927-34.

37. Shilton H, Gao Y, Nerlekar N, et al. Pre-operative bariatric clinic attendance is a predictor of post-operative clinic attendance and weight loss outcomes. Obes Surg. 2019;29(7):2270-5.

38. El Chaar M, McDeavitt K, Richardson S, et al. Does patient compliance with preoperative bariatric office visits affect postoperative excess weight loss? Surg Obes Relat Dis. 2011;7(6):743-8.

Publisher's Note Springer Nature remains neutral with regard to jurisdictional claims in published maps and institutional affiliations. 